\title{
A Challenging Criterion for Premature Ejaculation from a Wider Perspective: Evaluation with Intravaginal Ejaculatory Latency Time (IELT)
}

\author{
Ayse Veyhurda Dikmen* \\ Consultant Urologist, Polatlı Government Hospital, Turkey \\ *Corresponding author: Ayse Veyhurda Dikmen, Consultant Urologist, Polatli Government Hospital, Turkey
}

\begin{abstract}
ARTICLE INFO
Received: 幽 February 05, 2020

Published: 蔧 February 11, 2020

Citation: Ayse Veyhurda Dikmen. HA Challenging Criterion for Premature Ejaculation from a Wider Perspective: Evaluation with Intravaginal Ejaculatory Latency Time (IELT). Biomed J Sci \& Tech Res 25(3)-2020. BJSTR. MS.ID.004213.
\end{abstract}

Keywords: Premature Ejaculation; Intravaginal Ejaculation Latency Time (IELT); Male Sexual Dysfunctions; Ejaculation

\section{ABSTRACT}

Introduction and Objectives: Premature ejaculation has been a complicating issue for men ever after. IELT is a relatively simple and easy-to-understand method of evaluation of patients' response to treatment of premature ejaculation. We aimed to reassess the usability of this way of measurement of response to treatment of premature ejaculation.

Materials-Methods: 1600 male patients, aged between 21-92 (mean age: 42.3), who were diagnosed to have primary premature ejaculation in our urology clinic, with an intravaginal ejaculatory latency time (IELT) value below 1 minute, were evaluated after treatment retrospectively. IELT measurement and both choices of treatment, either daily or on-demand usage were told to patients' and their partners.

Results: 856 patients on daily treatment with citalopram $20 \mathrm{mg}$ daily had a pretreatment IELT between 45-60 seconds. Pretreatment IIEF scores of citalopram group were between 31-64 with mean IIEF score being $46.2 \pm 2.3(\mathrm{p}=0.043)$. Mean posttreatment IIEF score was $53.2 \pm 2.4(\mathrm{p}=0.023)$. Posttreatment mean IELT value was $165.2 \pm 12.1$ seconds, statistically significant $(\mathrm{p}=0.03)$. 670 patients on dapoxetine treatment had a pretreatment IELT below 60 seconds with a mean posttreatment IELT value of $187.3 \pm 13$.2seconds, statistically significant ( $p=0.028$ ). Pretreatment IIEF scores of dapoxetine group were between 31-67 with mean IIEF score being $44.5 \pm 2.8$ ( $p=0.039$ ). Posttreatment IIEF scores were between 36-68. Mean posttreatment IIEF score was 54.4 $\pm 2.6(\mathrm{p}=0.027)$.

Conclusion: Using IELT to evaluate premature ejaculation is a challenging but acceptable approach due to statistically significant outcomes in this retrospective study.

\section{Introduction}

In today's world of innovation and technology, every effort gets its correspondence in terms of reaching the best way of practice. Men's healthcare is also receiving its share from the ongoing prospective studies of diagnosis and treatment. When we consider the sexual life cycle, premature ejaculation has been a complicating issue for men ever after. It has been one of the most challenging problems in urology for ages. However, premature ejaculation, itself, has been a somehow subjectively measured complaint and a quite difficult issue to diagnose. Although men attend to urology clinic themselves when they suffer from premature ejaculation, it also bothers their female partners when the diagnosis and treatment were both taken into consideration. Since the history taking during urologic examination only depends on the male patient and the diagnosis is established according to his complaints, the situation can be IELT is a relatively simple and easy-to-understand method of evaluation of patients' response to treatment of premature ejaculation. Partners' attendance to measurement of IELT is a challenging approach to evaluate these patients' problems. We aimed to re-assess the usability of this way of measurement of response to treatment of premature ejaculation. 


\section{Material \& Methods}

1600 male patients aged between 21-92 (mean age: 42.3), who were diagnosed to have primary premature ejaculation in our urology clinic, with an intravaginal ejaculatory latency time (IELT) value below 1 minute, were evaluated after treatment retrospectively. The patients have undergone routine urologic evaluation and patients, who were diagnosed with urologic malignancies were excluded from the retrospective evaluation. The patients were given the medication, either dapoxetine or citalopram, which they preferred themselves. IELT measurement and both choices of treatment, either daily or on-demand usage were told to patients and their partners. For the process of measurement of IELT, the female partners were responsible for assessing the issues of using the stopwatch. Many urologists quite often ignore the inclusion of female partners into evaluation and assessment procedures when premature ejaculation is considered. However, sexual intercourse is a universal issue which necessitates the accountability of both partners in a scientific manner. So far, in tis respective evaluation, I have taken into account the couples only, in which the female partners have used the stopwatch and attended the duration of evaluation. Although it is somehow a confusing procedure for the female partners to use the stopwatch themselves and measure the IELT, no unexpected or unwanted situation were confronted in terms of female attendance for time-measuring. It is also quite surprising to observe the accurate outcomes of measurement during usage of stopwatch in sexual intercourse because there were lots of different types of female partners from different social and cultural layers of the society. No one of the female partners had difficulty of behaviour when they were told how to measure IELT and could accommodate themselves to the process.

\section{Results}

890 patients on daily treatment with citalopram $20 \mathrm{mg}$ daily had a pretreatment IELT between 45-60 seconds. 34 them stopped the medication early and were excluded. 856 patients on daily treatment with citalopram $20 \mathrm{mg}$ daily had a pretreatment IELT between 45-60 seconds. Pretreatment IIEF scores of citalopram group were between 31-64 with mean IIEF score being 46.2 \pm 2.3 $(\mathrm{p}=0.043)$. Posttreatment IIEF scores were between 37-65. Mean posttreatment IIEF score was $53.2 \pm 2.4(\mathrm{p}=0.023)$. Posttreatment IELT period of patients on daily citalopram for 12 weeks was found to be more than 150 seconds, with a mean IELT value of $165.2 \pm 12.1$ seconds, statistically significant $(p=0.03) .710$ patients were on dapoxetine $30 \mathrm{mg}$ on-demand treatment and 30 of these left treatment before and were excluded during retrospective evaluation. 670 patients on dapoxetine treatment had a pretreatment IELT below 60 seconds and posttreatment IELT period of patients on dapoxetine for 12 weeks was found to be more than 150 seconds, with a mean IELT value of $187.3 \pm 13.2$ seconds, statistically significant $(p=0.028)$. Pretreatment IIEF scores of dapoxetine group were between 31-67 with mean IIEF score being $44.5 \pm 2.8$ ( $p=0.039$ ). Posttreatment IIEF scores were between 3668. Mean posttreatment IIEF score was $54.4 \pm 2.6(\mathrm{p}=0.027) .10$ could not make a satisfactory self-evaluation.

\section{Conclusion}

Premature ejaculation has been one of the most çommon health problems of men lifelong [1]. Unexpected and uncontrolled ejaculation can be reversed with proper medications to reach the expected subsequent orgasm [1,2]. In fact, urologists, somehow, forget the accountability of both partners when premature ejaculation has been encountered as a prominent urologic issue. In some studies, it was detected that this item had been overestimated by nearly 1 minute [2]. However, the term of IELT is usually expected to differ between populations and even between different layers of socio-cultural ethnicity. Dapoxetine has been declared to be proven with its efficacy for acquired premature ejaculation [3]. So far in this retrospective study, the on-demand usage of dapoxetine was measured in terms of statistical significance for premature ejaculation treatment. Some authors have gone far to measure the probable associations between self-estimated IELT and symptom scores of prostatic diseases [4]. Although in some studies, it is declared that the decrased frequency of ejaculation and somehow the number of sexual intercourse that the subjects experience impair the mechanism of controlling over ejaculation [5], the patients in this study have not given a history of reduced frequency of premature ejaculation.

There is also a study which declares that premature ejaculation causes unwanted effects in terms of intimacy of couples and their relationship [6]. Since my study contains a large number of patients from different social and cultural layers of the population, it can provide a basis for a population nomogram and may reflect the state of affairs in whole population. There is also a study which determines the prevalance of premature ejaculation according to the changing norms of the population in a decade [7]. Such that, if we could only repeat the same retsopective study even a decade after with the same medications, perhaps new outcomes would reflect the current situation of the study population as well. As always, we need new studies, which are carried on in larger series of patients to determine premature ejaculation from the wider perspective of window. There are also some matters of fact that affect the course of ejaculation such as self-rating of ejaculation control and ejaculation-related distress [8,9]. One unexpected bias of this retrospective study could be the lack of measurement of testosterone in males but in a study by Morgentaler et.al. it was found that the estimated duration of ejaculation was not related with outcomes of measurement of serum testosterone [10].

As a respected proof of science, dapoxetine $30 \mathrm{mg}$ on-demand treatment was recommended as first line of treatment in a metaanalysis [11] and my study was one of its kind to compare the effects of both citalopram and dapoxetine with a large number of patients. 
Since premature ejaculation can affect quality of life of both men and their partners, we believe that it should be treated without any respect to age of the patients if they demand to be treated. IELT alone cannot be enough to evaluate the efficacy of treatment of premature ejaculation. Whether the patients and their partners have the correct and enough perception of this measure is the factor, affecting directly the success and follow-up of the treatment. Main factors, affecting the compliance to the medication, are the economic causes and the personal choices of intake, whether daily or on-demand. The results of this retrospective study are also valuable due to sociocultural factors since they reflected IELT being applied to different parts of population with premature ejaculation. Wider studies are needed to compare the efficacy of SSRIs and dapoxetine for treatment of premature ejaculation. Using IELT to evaluate premature ejaculation is a challenging approach due to sociocultural biases.

\section{Acknowledgment}

There is nothing to disclose. The author reports no conflicts of interest.

\section{References}

1. Salem AM, Kamel II, Rashed LA, Gamal El, Din SF (2016) Effects of paroxetine on intravaginal ejaculatory latency time in Egyptian patients with lifelong premature ejaculation as a function of serotonin transporter polymorphism. Int J Impot Res 29(1): 7-11.

2. Lee WK, Cho ST, Lee YS, Lee YG, Oh CY, et al. (2015) Can estimated intravaginal ejaculatory latency time be used interchangeably with stopwatch- measured intravaginal ejaculatory latency time for the diagnosis of lifelong premature ejaculation? Urology 85(2): 375-380.

ISSN: 2574-1241

DOI: $10.26717 /$ BJSTR.2020.25.004213

Ayse Veyhurda Dikmen. Biomed J Sci \& Tech Res

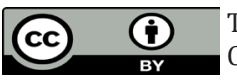

This work is licensed under Creative Commons Attribution 4.0 License

Submission Link: https://biomedres.us/submit-manuscript.php
3. Yue FG, Dong L, Hu TT, Qu XY (2015) Efficacy of Dapoxetine for the treatment of premature ejaculation: a meta-analysis of randomized clinical trials on intravaginal ejaculatory latency time, patient-reported outcomes, and adverse events. Urology 85(4): 856-861.

4. Zhang X, Tang D, Xu C, Gao P, Hao Z, et al. (2015) The relationship between self-estimated intravaginal ejaculatory latency time and International Prostate Symptom Score in middle-aged men complaining of ejaculating prematurely in China. J Sex Med 12(3): 705-712.

5. Palmieri A, Imbimbo C, Longo N, Fusco F, Verze P, et al. (2012) Ejaculatory abstinence influences intravaginal ejaculatory latency time: results from a prospective randomized trial. Urol Int 88(4): 459-462.

6. Abdo $\mathrm{CH}$ (2016) The impact of ejaculatory dysfunction upon the sufferer and his partner. Transl Androl Urol 5(4): 460-469.

7. Song WH, Yoo S, Oh S, Park J, Cho SY, et al. (2019) Ten-Year Interval Changes in the Prevalence of Self-Identified Premature Ejaculation and Premature Ejaculation Based on an Estimated Intravaginal Ejaculation Latency Time of $<3$ Minutes in the General Population: The Korean Internet Sexuality Survey (KISS) 2016. J Sex Med 16(4): 512-521.

8. Janssen PK, Waldinger MD (2016) The mathematical formula of the intravaginal ejaculation latency time (IELT) distribution of lifelong premature ejaculation differs from the IELT distribution formula of men in the general male population. Investig Clin Urol 57(2): 119-126.

9. Mc Mahon C, Althof S, Rosen R, Giuliano F, Miner M, et al. (2019) The Oxytocin Antagonist Cligosiban Prolongs Intravaginal Ejaculatory Latency and Improves Patient-Reported Outcomes in Men with Lifelong Premature Ejaculation: Results of a Randomized, Double-Blind, PlaceboControlled Proof-of-Concept Trial (PEPIX). J Sex Med 16(8): 1178-1187.

10. Morgentaler A, Polzer P, Althof S, Bolyakov A, Donatucci C, et al. (2017) Delayed Ejaculation and Associated Complaints: Relationship to Ejaculation Times and Serum Testosterone Levels. J Sex Med 14(9): $1116-1124$

11. Jian Z, Wei X, Ye D, Li H, Wang K (2018) Pharmacotherapy of premature ejaculation: A systematic review and network meta-analysis. Int Urol Nephrol 50(11): 1939-1948.

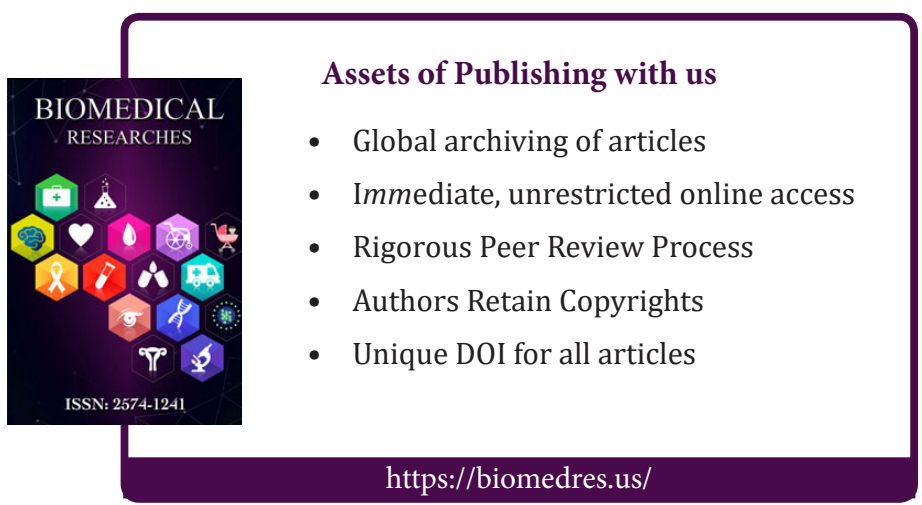

Copyright@ Ayse Veyhurda Dikmen | Biomed J Sci \& Tech Res | BJSTR. MS.ID.004213. 